Learning correspondences between magnitudes, symbols and words: Evidence for a triple code model of arithmetic development

\author{
Stephanie A. Malone \\ Michelle Heron-Delaney \\ Australian Catholic University
}

Kelly Burgoyne

University of Manchester

and Australian Catholic University

\author{
Charles Hulme \\ University of Oxford \\ and Australian Catholic University
}

Author Note:

This research was supported by a grant from the Australian Catholic University.

Correspondence concerning this manuscript should be addressed to Charles Hulme

Email: charles.hulme@education.ox.ac.uk 


\begin{abstract}
According to the Triple Code Model, early arithmetic development depends on learning the mappings between non-verbal representations of magnitude (quantity) and symbolic verbal (number words) and visual (Arabic numerals) representations of number. We examined this hypothesis in a sample of 166 4- to 7-year old children. Children completed 4 paired-associate learning tasks and a broad range of measures assessing early numerical (symbolic and non-symbolic magnitude comparison, digit writing, arithmetic) and reading skills (letter-sound knowledge, phoneme awareness, rapid automatized naming, word reading). A path model showed that paired-associate learning tasks involving mapping magnitudes onto verbal or visual stimuli predicted arithmetic performance over and above other well-established predictors. This relationship was specific to arithmetic and, consistent with the Triple Code Model, highlights that mapping between non-symbolic magnitude representations and the corresponding symbolic forms (verbal and visual) is important to the development of arithmetic skills.
\end{abstract}

Key words: Paired-associated learning, Triple Code Model, Approximate Number System (ANS), arithmetic development, numerical cognition, non-symbolic magnitude judgment 


\section{Learning correspondences between magnitudes, symbols and words: Evidence for a triple code model of arithmetic development}

The Triple Code Model (Dehaene \& Cohen, 1995) posits that numerical processing involves three categories of mental representation: two symbolic forms in which numbers are represented as words (e.g., “three”) and Arabic numerals (e.g., “3”) and a non-symbolic analogue system which represents numbers as magnitudes (quantities). These three classes of representation are seen as separate but linked, and an important requirement in early arithmetic development is to learn the mappings between them. Thus, according to this model, deficits in developing any of the three classes of representation (number words, Arabic numerals, magnitudes), or problems learning the mappings between them, might lead to difficulties in arithmetic.

Learning the mappings between number words, Arabic numerals and nonverbal magnitude representations is a clear example of paired-associate learning. Paired-associate learning is the ability to form connections between arbitrary pairs of stimuli such as visual symbols and their verbal labels (Litt, de Jong, van Bergen \& Nation, 2013). The ability to form such mappings is known to predict reading ability (Litt \& Nation, 2014; Clayton, Sears, Davis, \& Hulme, 2018; Warmington \& Hulme, 2012) but the extent to which paired-associate learning predicts arithmetic skills is as yet unclear. In this paper we explore whether differences in paired-associate learning (specifically learning mappings between magnitudes and verbal labels or visual symbols) are related to individual differences in children’s arithmetic skills.

\section{Non-symbolic magnitude representation and arithmetic skills}

Rudimentary non-symbolic representations of magnitude are present in early infancy (Xu \& Spelke, 2000) and are associated with visual and phonological 
representations of number during language acquisition (von Aster \& Shalev, 2007; but see Lyons, Bugden, Zheng, De Jesus, \& Ansari, 2018). The Triple Code Model suggests that children's understanding of magnitudes (coded in the approximate number system; ANS) is important as it provides the semantic underpinning for Arabic numeral and number word representations (Dehaene \& Cohen, 1995); this idea has been supported by both computational modeling and neuroimaging studies (Lyons \& Ansari, 2009; Verguts \& Fias, 2004). In this view the ANS provides a critical foundation for the development of arithmetic skills. Recent meta-analyses report a modest relationship between performance on non-symbolic magnitude judgement tasks (a measure of the ANS) and arithmetic performance ( $r$ s = .20-.24; Chen \& Li, 2014; Fazio, Bailey, Thompson, \& Siegler, 2014; Schneider et al., 2017; but see De Smedt, Noel, Gilmore, \& Ansari, 2013 who report only a weak relationship). It has also been argued that an impairment in the processing of magnitudes is a central deficit in mathematics disorder (i.e. deficit in representing magnitudes: Butterworth, 2010; Piazza et al., 2010; or accessing magnitude representations: Rouselle \& Noel, 2007).

\section{Number knowledge and arithmetic skills}

A variety of evidence shows that children's number knowledge (knowledge of Arabic numerals and spoken number words) is predictive of arithmetic attainment. Numerosity judgements with Arabic numeral pairs (identifying the larger number in a pair of Arabic numerals) are more strongly related to arithmetic ability than analogous non-symbolic magnitude comparison tasks (e.g., Holloway \& Ansari, 2009; Rousselle \& Noel, 2007; Schneider et al., 2017). In addition, children who experience difficulties in arithmetic are slower to count dots (Landerl, Bevan, \& Butterworth, 2004), and name digits, but not letters or geometric shapes, than typically developing 
children (van de Sluis, de Jong, \& van der Leij, 2004; Pauly et al., 2011). This indicates that their difficulties are specific to words linked to magnitude representations. Furthermore, Göbel, Watson, Lervag and Hulme (2014) showed that knowledge of Arabic numerals at 6-years uniquely predicted arithmetic skills at 7years. These findings suggest that symbolic number knowledge is critical for the development of early arithmetic ability; a finding which is further highlighted by Martin, Cirino, Sharp and Barnes (2014) who demonstrated that symbolic number knowledge predicted variations in mathematics performance, above and beyond counting ability.

\section{Mapping representations of number and arithmetic}

Given the apparent importance of associations between different representations of number (Dehaene \& Cohen, 1995), research has begun to explore how children's skills in mapping between different representations of number relate to their early arithmetic performance. Mundy and Gilmore (2009) and Brankaer, Ghesquière and De Smedt (2014) assessed the ability of 6- to 8-year-olds to map between symbolic representations of number (Arabic numerals and spoken words) and their corresponding magnitudes (e.g. map the digit 8 to a set of eight dots); Libertus, Odic, Feigenson and Halberda (2016) examined younger children's (5- to 7years-old) ability to map between magnitudes and exact number words. These studies found that children's mapping ability accounted for individual differences in arithmetic performance, over and above other known predictors (e.g. non-symbolic and/or symbolic number comparison). The critical role of cross-modal pairing has also been highlighted by Defever, De Smedt and Reynvoet (2013): when asked to determine whether two stimuli depicted matching numerosities, children with mathematical learning difficulties were slower than typically developing children to 
respond to cross-modal pairs (matching a dot array to a corresponding Arabic numeral; 5 dots vs. “5”). In contrast, the two groups did not differ in the speed of responding on a comparable within-modal task (matching two dot arrays).

Extending this research, Hurst, Anderson and Cordes (2017) examined 3- to 4year-olds ability to map between the three aspects of the Triple Code Model: numeralto-magnitude, word-to-magnitude, and numeral-to-word. Like Brankaer et al. (2014), they found that performance was lowest when mapping between quantities and their associated symbols. They suggested that children may first map numerals to number words and only through this mapping, subsequently learn the mapping between numerals and the quantities they represent.

Taken together, these earlier studies show that children's knowledge of the associations between different representations of number is related to arithmetic ability (Brankaer et al., 2014; Defever et al., 2013; Libertus, et al., 2016; Mundy \& Gilmore, 2009). Furthermore, they demonstrate that pairings which involve nonverbal numerosities are often difficult for children (Brankaer et al., 2014; Hurst et al., 2017). These studies, however, all examine previously learned associations between quantities and Arabic numerals/number words. In the current study we examine the acquisition of these mappings and the extent to which this ability relates to arithmetic.

\section{Paired-associate learning and arithmetic skills}

Learning the mappings between alternative representations of number is a form of paired-associate learning. Only one study to date has directly investigated the relationship between paired-associate learning and arithmetic. Cui et al. (2017) found no significant relationship between visual-verbal paired-associate learning and addition and subtraction ability in 5- to 6-year-old Chinese children. Their visualverbal paired-associate learning condition required children to learn pairings between 
spoken nonsense words and pictures of imaginary animals. However, the Triple Code Model (Dehaene \& Cohen, 1995) suggests that it is learning the associations between nonverbal magnitudes and verbal labels or visual symbols that may be particularly crucial for arithmetic development. No prior studies have investigated this possibility; the current study will fill this gap.

\section{Current study}

This study examines the role of paired-associate learning as a predictor of arithmetic in 4.5- to 7-year-old children. We tested children on four paired-associate learning conditions using verbal (nonsense words), visual (abstract symbols) and magnitude stimuli (dot arrays). There were four conditions: magnitude-verbal, magnitude-visual, visual-verbal, and verbal-visual. Children also completed measures of formal arithmetic (addition), symbolic and non-symbolic magnitude understanding (as assessed using digit and dot comparison tasks) and number knowledge (digit writing). In line with the theory that magnitude representations (coded in the ANS) are a critical foundation for symbolic number knowledge and arithmetic (Dehaene and Cohnen, 1995; Piazza \& Dehaene, 2004), we predicted that paired-associate learning will only be related to arithmetic ability in the conditions involving learning associations with magnitudes (i.e., magnitude-visual; magnitude-verbal).

The relationship between paired-associate learning and reading is used as a control condition to investigate whether paired-associate learning involving magnitudes is specifically related to arithmetic. Paired-associate learning tasks that involve a verbal output are known to be correlates of reading ability (Clayton, Sears, Davis \& Hulme, 2018; Litt \& Nation, 2014) so we predict that paired-associate 
learning conditions involving a verbal output (i.e., visual-verbal; magnitude-verbal) will be correlates of reading performance.

\section{Method}

\section{Participants}

Participants were 166 children from a primary school in Brisbane, Australia (81 boys; mean age $=70.62$ months, range $54-87$ months, $S D=7.34$ ). Children were in their first (Preparatory Year; $n=94$, mean age $=65.30, S D=3.99$ ) or second year of school (Grade $1 ; n=72$, mean age $=77.57, S D=4.23$ ). The Australian Catholic University Human Research Ethics Committee (2015-141H) provided ethical approval, parental consent was obtained using opt-out consent and children provided verbal assent prior to participation. The sample was unselected for ability; all children who consented to participate were included.

\section{Materials and Procedure}

Children completed a range of tasks individually in 4 testing sessions administered over 4 weeks (one session per week).

Paired-associate learning tasks. Children completed four paired-associate learning conditions (one condition per week) in a randomized order: magnitudevisual, magnitude-verbal, visual-verbal, and verbal-visual. The stimuli used were magnitudes (random dot arrays), abstract visual symbols (letter like forms) and spoken nonwords. There were four stimulus-response pairs per condition. These stimulus-response pairs were fixed within conditions meaning all children were exposed to the same pairs (see Figure 1). The order of presentation of these pairs in each condition was randomized. 
Each condition followed the same structure: two practice blocks (four trials per block) followed by five learning/test blocks (four trials per block). Stimuli were presented on small square cards $\left(5 \mathrm{~cm}^{2}\right)$. During the practice trials, children began learning the stimulus-response mappings. In the first practice block, children were exposed once to each stimulus-response pair and encouraged to copy each response either by drawing it in a response booklet (symbol output conditions; magnitudevisual and verbal-visual) or by saying it (verbal output conditions: magnitude-verbal and visual-verbal). During the second practice block, children were asked to focus on learning which stimulus was associated with which response, instead of actively reproducing the response. Feedback was provided as necessary throughout the practice blocks. The practice blocks lasted approximately two to three minutes.

Following the practice blocks, children received five learning/test blocks. As is standard in experiments of this sort (e.g., Warmington \& Hulme, 2012), the number of correct responses across all learning/test blocks was used as a measure of learning. During each learning/test block children were shown each of the stimuli individually in a random order and asked to provide the associated response (i.e. "What symbol/word goes with this?”). Regardless of the child's response, the experimenter provided the correct answer. Each correct response produced by the child received a score of 1 . A verbal response was scored as correct if all phonemes were spoken correctly; a symbol response was interpreted as correct if it contained all the features of the target symbol and was recognizable out of context. The maximum score per block was 4 (one per stimulus-response pairing) giving a maximum possible score of 20 for each condition.

Visual (symbol) stimuli. Three sets of four symbols were taken from extinct languages (see Figure 1). A pilot study was completed to assess the ease with which 
the symbols could be drawn and labeled. Fifteen adults ( 4 male; mean age $=36.13$ years; $S D=15.22$ ) were presented with 50 symbols and asked to draw each symbol and report on a 10-point scale how easy it was to draw ( 1 = very easy; $10=$ very difficult) and to name ( 1 = very easy; 10 = very difficult). An average difficulty score for ease of drawing and naming was calculated for each symbol.

Symbols with an average naming rating of 4 or greater, and a drawing rating of 5 or less were selected. This gave symbols that were relatively easy to draw and for which children were unlikely to have idiosyncratic labels. The 12 symbols were divided across the three conditions, matching difficulty ratings for the symbols across conditions.

Verbal (nonword) stimuli. Three sets of four consonant-vowel-consonant nonwords were used (one set for each condition that included verbal stimuli). To ensure that each word was distinct within a condition, no two words in a set began or ended with the same phoneme or contained the same vowel. Nonword stimuli sets were (1) nid, dut, faz, pel; (2) mab, tup, rin, zom; (3) fet, dax, vok, wug.

Magnitude stimuli. Eight dot arrays were used (four per condition: magnitudeverbal and magnitude-visual). As it was necessary for children to discriminate easily between the arrays within a condition, a ratio of 1:2 was applied between the numerosities (Halberda \& Feigenson, 2008). The numerosities varied from 6 to 56 (set 1: 6, 12, 24 and 48; set 2: 7, 14, 28 and 56), with the lowest value being greater than the subitizing range, therefore ensuring that the approximate number sense was activated (Bonny \& Lourenco, 2013). Each child was only presented with the numerosities from one set per condition.

The dot arrays consisted of black dots of varying sizes on a white background. The surface area of each array was constant so that responses had to be based on 
numerosity rather than surface area. This resulted in the size of the dots varying depending upon the specific magnitude. Critically, to ensure that the children were learning the magnitude rather than the pattern of dots, the dots were also presented in a different spatial configuration on each trial. Thus, each magnitude within a condition was associated with seven presentation arrays: two for use during practice blocks, and five for learning blocks.

\section{Measures of cognitive ability.}

Alphabetic skills which form the foundation for learning to read were assessed using two subtests of the York Assessment of Reading Comprehension (Hulme et al., 2009). Phoneme deletion was measured using the sound deletion subtest. In this test children were asked to delete sounds from 12 spoken words (e.g., "Say sheep without the "p” sound”). The letter-sound knowledge (core) test examined children's ability to produce the sound associated with 17 letters or digraphs.

Rapid automatized naming (RAN) was used as a measure of processing speed. There were 2 RAN tasks: colours (red, green, black, blue, brown) and pictures (eye, dog, key, lion, table). Four trials were completed (2 x RAN colours and $2 \times$ RAN pictures). For each trial, children were presented with an 8 x 5 item matrix displaying 8 repetitions of the items in a random order. Children were asked to name each of the stimuli as quickly as possible; time taken to name the complete set of items was recorded. RAN rate for each trial was calculated: number correct (max. 40)/response time.

Digit Writing. Children were asked to write ten numbers $(2,7,13,28,69,145$, $314,648,1052,2114)$ read aloud by the experimenter. One point was awarded for each correct response (reversals of digits were scored as errors). 
Digit Comparison (Test of Basic Addition and Number Skills (TOBANS):

Hulme, Brigstocke, \& Moll, 2016). This subtest assessed symbolic magnitude understanding. Children were presented with pairs of single digits printed in a $21 \mathrm{x}$ 29.7cm booklet. Stimulus pairs (48 in total) were presented in two columns with 8 pairs per column. Numbers ranged from 1 to 9, with the difference between the numerosities in each pair ranging from 1 to 8 . Children were asked to select the more numerous stimulus in each pair. They were given 30 seconds to complete as many items as possible. One point was awarded per correct answer.

Nonsymbolic magnitude comparison. Three conditions which differed in the ratio of the magnitudes represented were completed in a fixed order from the most difficult condition to the easiest: Ratio 0.86 , Ratio 0.66 and Ratio 0.57 . This order was used to reduce children's frustration with the task as they progressed through the conditions. All conditions were presented in a $14.8 \times 21 \mathrm{~cm}$ booklet, with six stimulus pairs displayed per page (one per row). Each pair consisted of two arrays of small black dots displayed in $2.2 \mathrm{~cm}^{2}$ boxes, with the arrays being equated for surface-area to ensure that this could not serve as an additional cue to numerosity. The numerosities of the arrays varied from 6 to 11 in the Ratio 0.86 condition, 21 to 45 in the Ratio 0.66 condition, and 5 to 13 in the Ratio 0.57 condition.

Before each condition there was a set of six practice stimulus pairs which children completed without time constraints. This was followed by the test proper: children were informed that they had 30 seconds to tick the more numerous array in as many stimulus pairs as possible. They were also told that they should not count the arrays, since that would be too slow. There were 30 pairs per Ratio condition (90 pairs in total). These measures are similar to those used by Nosworthy, Bugden, Archibald, 
Evans and Ansari (2013) and essentially measure the "efficiency" of the ANS (cf. Goebel et al. 2014). One point was awarded per correct answer.

Non-Verbal IQ was assessed using Raven’s Coloured Progressive Matrices (Raven, Court, \& Raven, 1986). Children were presented with 3 practice items and 12 test items. For each item, a puzzle was presented with a piece missing. Children selected which of six potential pieces could complete the puzzle.

\section{Measures of attainment.}

Word Reading Fluency. Children were given a list of 144 single words graded in difficulty and asked to read as many as they could in 1 minute. Reading fluency was calculated: (total words correct)/60 (seconds).

Arithmetic (addition). In an untimed task, children were presented with up to 20 printed addition questions which were read aloud by the examiner. The first ten items consisted of single-digit addends (summing less than 10). The final ten items consisted of single-digit addends totaling greater than 10 . Suitability of these items for children of this age was determined by consulting a focus group of teachers, who confirmed that these sums were similar in difficulty level to those taught as part of the curriculum for this age group. All children were required to attempt the first ten items. For the latter 10, testing was discontinued after 3 consecutive incorrect responses. Children provided their responses verbally.

The order of the four testing sessions was counterbalanced, however the task order within a given session was fixed, so that the PAL task was always administered first due to it being the longest and most taxing task. The ordering of the tasks within each session was as follows: (1) Magnitude-visual PAL, YARC phoneme deletion, Addition task; (2) Magnitude-verbal PAL, RAN; (3) Visual-verbal PAL, Raven’s matrices, Nonsymbolic magnitude comparison, Digit comparison, Reading; (4) 
Verbal-visual PAL; YARC letter sound knowledge and Digit writing. Each session was approximately 30 minutes in duration. In addition to the tasks reported, other cognitive tasks were administered, which are not relevant to the current study, and therefore not reported in this paper.

\section{Results}

The means, standard deviations, reliabilities and measures of skewness, and kurtosis and for all variables are shown in Table 1 (all data are available on the OSF website; Malone, Heron-Delaney, Burgoyne, \& Hulme, 2016). Correlations between variables are shown in Table 2. Most of the known predictors of reading and arithmetic correlate in the expected direction, with the exception of magnitude comparison. For these, the correlations were slightly stronger between magnitude comparison conditions and reading than magnitude comparison conditions and arithmetic. The difference in strength of the correlations between each magnitude condition (.86, .66, and .57) and either reading or arithmetic were compared using a test of the difference between related correlations (Fisher's z). None of the differences in the size of these pairs of correlations between magnitude comparison and reading/math were significant $(\mathrm{zs}=.67-1.46, \mathrm{ps}>.05)$.

Performance on the paired-associate learning tasks showed a good range of scores (verbal output: 26.3 to 34\% correct; visual output: 48.6 to $54.55 \%$ correct), with the verbal-output conditions being harder. Levels of performance here are comparable to previous research examining children’s paired-associated learning (e.g., Hulme et al., 2007: verbal output $=20.56$ to 35.4\%; visual output $=53.76 \%$; Litt et al., 2013: verbal output $=16.2$ to $19.47 \%$; visual output $=33.37$ to $38.7 \%$ ). Furthermore, although it has been previously demonstrated that children have difficulty mapping between non-symbolic and symbolic representations of number 
(e.g., Brankaer et al., 2014; Mundy \& Gilmore, 2009), their accuracy in the magnitude conditions (verbal output: 26.3\%; visual output: 48.6\%) was slightly lower though similar to the non-magnitude conditions (verbal output: 34\%; visual output: 54.44\%). The two magnitude paired-associate conditions (magnitude-visual; magnitude verbal) correlate better with addition than the two non-magnitude conditions (visual-verbal; verbal-visual). However, the two magnitude pairedassociate conditions also show substantial correlations with reading, the correlation being particularly strong for the magnitude-verbal condition (which is expected to correlate with reading because it involves learning a verbal (spoken) response).

To explore the unique relationships between our different cognitive abilities, paired-associate learning and measures of arithmetic and reading, a path analysis (simultaneous regression model) was conducted (see Figure 2) using MPlus 8.1 (Muthén \& Muthén, 1998-2017). Missing values (n = 6) were handled with Full Information Maximum Likelihood estimation. All variables in this model were first regressed on age and non-verbal IQ, thus the model represents relationships between variables that are independent of the shared variance attributable to IQ and age. For the sake of simplicity these regressions are not shown in the model. Following this, addition and reading were regressed on all the predictors. Non-significant regression paths and non-significant covariances between predictors were dropped iteratively, resulting in the simplified model shown in Figure 2 in which all relationships are statistically significant. The covariances between predictors that were retained in the model are shown in Table 3.

In this model arithmetic is predicted by the two paired-associate learning conditions that involved learning mappings to magnitude representations together with digit comparison (a measure of symbolic magnitude understanding), digit 
writing, and letter-sound knowledge. In contrast, for reading, only the pairedassociate learning conditions involving verbal-output (visual-verbal and magnitudeverbal) were unique predictors over and above the other well-established predictors of reading (i.e., phoneme deletion, letter-sound knowledge and RAN). Digit writing was also a predictor of reading. Our results therefore show convincingly that pairedassociate learning involving magnitudes is specifically related to arithmetic. In addition, in line with earlier findings, paired-associate learning tasks involving a spoken response are unique predictors of reading ability (Litt et al., 2013; Litt \& Nation, 2014; Clayton et al., 2018).

Overall, the model accounts for $57 \%$ of the variance in arithmetic and $73 \%$ of the variance in reading and provides a very good fit to the data $\left(\chi^{2}(45)=61.23, p=\right.$ 0.05, Root Mean Square Error of Approximation $(\mathrm{RSMEA})=.05(90 \% \mathrm{CI}=0.000$ 0.075), Comparative Fit Index $(\mathrm{CFI})=.99)$.

\section{Discussion}

This study examined the associations between different forms of pairedassociate learning and variations in arithmetic skills in young children. As hypothesised, paired-associate learning involving mappings between magnitudes and verbal labels or visual symbols predicted individual differences in arithmetic skills in children, even after controlling for the effects of other established predictors of arithmetic. In contrast, paired-associate learning tasks involving mappings with verbal output (visual-verbal; magnitude-verbal) predicted reading ability, consistent with previous research (e.g., Litt and Nation, 2014; Clayton et al., 2018). In short, our findings show that learning the association between magnitude representations and their corresponding symbolic form is particularly relevant to arithmetic performance, 
while paired-associate learning tasks involving verbal (spoken) outputs are a predictor of reading ability.

\section{A triple code theory of arithmetic development}

We have shown that children who are better at forming associations between non-symbolic magnitudes and either visual symbols or verbal labels are better at arithmetic, even after controlling for several other well-established predictors of arithmetic ability. This finding is novel and provides support for the Triple Code Model (Dehaene \& Cohen, 1995) and its relevance to the development of arithmetic skills. The Triple Code Model is based on adult data, primarily from neuropsychological patients who show impairments in arithmetic following brain damage. According to the model, number knowledge involves three categories of mental representation: number words, Arabic numerals, and an analogue magnitude representation system. Critically, these three separate classes of representation are linked. A direct implication of the model is that the mappings between magnitudes, number words and Arabic digits need to be learned. The creation of such mappings must logically depend upon paired-associate learning mechanisms. Our data provide support for the importance of learning arbitrary mappings between non-symbolic representations of magnitude and symbolic representations (visual and verbal stimuli) for arithmetic development.

Our findings align with earlier studies showing that the ability to map between non-symbolic magnitudes and visual and verbal labels is related to arithmetic (e.g., Brankaer et al. 2014; Libertus et al., 2016; Mundy \& Gilmore, 2009). It is important to emphasise however, that these studies assessed children's ability to use previously learned associations between quantities and Arabic digits/number words. We have 
extended these findings by demonstrating that variations in the ability to acquire these mappings are associated with arithmetic ability. The absence of a relationship between visual-verbal paired-associate learning and arithmetic in our study is consistent with the pattern reported by Cui et al. (2017).

The learning of associative links between magnitudes and nonwords in the present study can be seen as analogous to learning to relate magnitudes to number words. Similarly, learning to link magnitudes and symbols can be seen as analogous to learning magnitude-Arabic numeral mappings. This reflects the observation that Western language systems were created with the specific intention of communicating exact numbers that lie beyond the subitizing range (Núñez, 2017). Theoretically, our findings suggest that variations in children’s ability to learn magnitude-verbal mappings and magnitude-symbol mappings may be one determinant of variations in arithmetic skills. More speculatively, this aligns with the idea that children’s understanding of non-symbolic magnitude representations is important as it provides the semantic underpinning for Arabic numeral and number word representations (Dehaene \& Cohen, 1995) and that an impairment in the processing of numerosities may be a central deficit in mathematics disorder (e.g., Butterworth, 2010; Piazza et al., 2010).

When interpreting our findings concerning learning about magnitudes we must adopt a degree of caution as continuous perceptual features (e.g. cumulative surface area and convex hull; see Dietrich, Huber, \& Nuerk, 2015; Gebuis \& Reynvoet, 2011; Leibovich, Katzin, Harel, \& Henik, 2017; Merkley \& Scerif, 2015) may serve as additional cues in making judgements about numerosities. In the natural environment a correlation exists between numerosity and perceptual features, such as surface area (Leibovich et al., 2017). For example, a larger number of fruits will involve a larger 
surface area than a smaller number. While total surface area was controlled for in the arrays used here, other visual properties, such as total perimeter and density, were not, and it is possible that some combination of other cues was positively correlated with numerosity. However, Gebuis and Reynvoet (2012) have argued that such effects are unavoidable. We decided to control for total surface area since this has been shown to be the most salient visual cue to numerosity in young children (Rousselle et al., 2004). Nevertheless, it remains possible that other perceptual cues (De Smedt, Noel, Gilmore, \& Ansari, 2013) may have provided cues to numerosity (e.g. convex hull) in the current study. Future studies are needed to investigate whether controlling for these features influences the relationship between magnitude paired-associate learning and arithmetic performance.

Our findings are also consistent with previous research showing the importance of children's number knowledge (e.g., knowledge of Arabic numerals) for arithmetic performance (e.g., Gobel et al, 2014; Schneider et al., 2017; van de Sluis et al., 2004). As expected, we found that symbolic numerosity discrimination (digit comparison) was a stronger correlate of arithmetic ability $(r=.45)$ than non-symbolic numerosity discrimination $(r s=.18-.28)$. This finding replicates a number of earlier studies (Schneider et al., 2017; Xenidou-Dervou, Molenaar, Ansari, van der Schoot, \& van Lieshout, 2017). It is likely that the large proportion of common variance between digit and magnitude comparison tasks $(r=.63)$ accounts for why magnitude comparison is not a unique predictor of arithmetic in the current study. Finally, digit writing was also a correlate of arithmetic ability in this study, in line with earlier findings (e.g. Johansson, 2007).

Somewhat unexpectedly, letter-sound knowledge was a significant predictor of arithmetic in this study (as well as a predictor of reading, as expected). Letter- 
sound knowledge and digit writing were highly correlated $(r=.69)$ and both measures predicted both arithmetic and reading. Our interpretation is that both digit writing and letter-sound knowledge reflect different aspects of a child's knowledge of the association between visual symbols and verbal labels. It is well established that letter-sound knowledge is a critical foundation for learning to read (e.g. Hulme \& Snowling, 2012), while analogously, learning arithmetic depends on knowledge of Arabic numerals which is one of the strongest predictors of children's arithmetic skills (Gobel et al, 2014). In short, both reading and arithmetic depend upon learning symbol sets (letters or Arabic digits respectively) which appear to depend, at least in part, on some common cognitive processes.

\section{Alternative perspectives and future directions}

The nature of the relationship between non-symbolic and symbolic magnitude understanding and arithmetic development is currently a topic of intense debate. The number sense account (Dehaene, 1997) suggests that the integrity of a preverbal nonsymbolic magnitude system is critical as a foundation for the development of symbolic number knowledge, which in turn provides a foundation for the later development of formal arithmetic. In contrast, others have suggested that knowledge of the symbolic number system supports the development of non-symbolic number skills. Specifically, it has been suggested that children first learn the count sequence by rote, with these numbers serving as placeholders onto which non-symbolic magnitudes can be mapped (Carey, 2009; Wiese, 2003). So, for example, Lyons et al. (2018) found that kindergarteners' symbolic number knowledge was predictive of later non-symbolic number knowledge, and Merkley, Shimi and Scerif (2016) demonstrated that adults can learn a novel sequence of symbols (akin to rote counting) and later associate these with representations of magnitudes. 
We believe our findings are consistent with the number sense account (Dehaene, 1997; Dehaene \& Cohen, 1995), insofar as we have shown that children's ability to learn mappings between visual symbol or nonword responses and stimulus magnitudes was associated with variations in children's arithmetic. In our analyses we controlled for concurrent symbolic and non-symbolic number knowledge, as predictors of arithmetic, so we can be relatively certain that the ability to associate non-symbolic magnitudes with their symbolic counterparts is uniquely predictive of arithmetic. The data we have presented, however, are concurrent and therefore do not provide any strong evidence for a causal relationship. It would be particularly interesting to extend the current study to a longitudinal design and to examine the extent to which early variations in the PAL tasks used here (measured preferably in children aged around 4 years of age) are predictive of later progress in arithmetic (assessed after a year or so of formal instruction in arithmetic). Furthermore, it is important to emphasize that there may be bidirectional causal relationships operating: early variations in an ANS magnitude system may be causally related to learning symbolic number skills, while conversely, early emerging symbolic number skills may feedback and serve to refine the ANS magnitude system. Longitudinal studies, with multiple measures of these constructs are required to tease apart such potentially bidirectional influences.

Given that the acquisition of symbolic number knowledge begins prior to formal schooling, it would be of interest to examine how paired-associate learning relates to early number knowledge in pre-school children. Although the ability to learn paired associates has been examined to some extent within this age group, much of this research explores previously known numbers (e.g. Hurst et al., 2017). One exception to this is Carey, Shusterman, Haward and Distefano (2017) who taught 
three-knowers (i.e. children who understand the numbers 1, 2 and 3 only) the meaning of the number 4 and 10. After training, children could determine which of two magnitude arrays represented 4 items (e.g. 4 vs. 16), but had difficulty in selecting the array containing 10 items (e.g. 10 vs. 30). Test trials for the number 10 also revealed no evidence of a ratio effect, which was interpreted as evidence that magnitude representations do not underpin the meaning of number words in early development. Given that our analysis demonstrated magnitude paired-associate learning conditions to be related to variations in arithmetic during the early stages of formal schooling, it would be of interest to examine the developmental trajectory of this relationship starting with younger children.

\section{Reading development}

We used reading as a control outcome measure so we could demonstrate specificity in the skills associated with arithmetic development. Our findings confirm the results from many previous studies showing that phoneme awareness, letter-sound knowledge and Rapid Automatized Naming are critical and powerful predictors of variations in reading development (Hulme \& Snowling, 2012, 2013). Furthermore, consistent with previous studies (Litt \& Nation, 2014; Clayton et al., 2018), we found that paired-associate learning tasks that involve a verbal output account for variance in reading performance. This pattern is in line with the suggestion of Clayton et al. (2018) who argued that the amount of phonological information needed to be learned in a paired-associate learning task is a critical influence on the strength of the relationship between paired-associate learning and reading. The tasks used here involved learning a spoken nonword response and place substantial emphasis on learning phonological information (although less demands than learning to associate pairs of spoken nonwords, as in the Clayton et al. study). 


\section{Conclusion}

This study is the first to investigate whether differences in paired-associate learning ability (specifically, learning associations between magnitudes and verbal labels or visual symbols) are related to individual differences in arithmetic in children. Children who were better at learning mappings between magnitudes and verbal labels or visual symbols also demonstrated better arithmetic skills, even after controlling for a range of better established correlates of arithmetic. Our findings have important theoretical implications and provide evidence for a Triple Code Model of arithmetic development. In such a view, learning the mappings between magnitudes, number words and Arabic numerals provides the foundation for symbolic number skills, which in turn are critical for the development of formal arithmetic skills. 


\section{References}

Bonny, J. W., \& Lourenco, S. F. (2013). The approximate number system and its relation to early math achievement: Evidence from the preschool years. Journal of Experimental Child Psychology, 114, 375-388. doi:

10.1016/j.jecp.2012.09.015

Brankaer, C., Ghesquière, P., \& De Smedt, B. (2014). Children’s mapping between non-symbolic and symbolic numerical magnitudes and its association with timed and untimed tests of mathematics achievement. PLoS One, 9. doi: 10.1371/journal.pone.0093565

Butterworth, B. (2010). Foundational numerical capacities and the origins of dyscalculia. Trends in Cognitive Sciences, 14, 534-541. doi:10.1016/j.tics.2010.09.007

Carey, S. (2009). Where our number concepts come from. Journal of Philosophy, 106(4), 220-254.

Carey, S., Shusterman, A., Haward, P., \& Distefano, R. (2017). Do analog number representations underlie the meanings of young children's verbal numerals? Cognition, 168, 243-255. doi: 10.1016/j.cognition.2017.06.022.

Chen, Q., \& Li, J. (2014). Association between individual differences in nonsymbolic number acuity and math performance: A meta-analysis. Acta Psychologia, 148, 163-172. doi: 10.1016/j.actpsy.2014.01.016

Clayton, F., Sears, C., Davis, A., \& Hulme, C. (2018). Verbal task demands are key in explaining the relationship between paired-associate learning and reading ability Journal of Experimental Child Psychology, 171, 46-54. doi: 10.1016/j.jecp.2018.01.004 
Cui, J., Georgiou, G. K., Zhang, Y., Li, Y., Shu, H., \& Zhou, X. (2017). Examining the relationship between rapid automatized naming and arithmetic fluency in Chinese kindergarten children. Journal of Experimental Child Psychology 154, 146-163. doi: 10.1016/j.jecp.2016.10.008

De Smedt, B., Noël, M-P., Gilmore, C., Ansari, D. (2013). How do symbolic and nonsymbolic numerical magnitude processing skills relate to individual differences in children's mathematical skills? A review of evidence from brain and behavior. Trends in Neuroscience and Education, 2, 48-55. doi: 10.1016/j.tine.2013.06.001

Defever, E., De Smedt, B., \& Reynvoet, B. (2013). Numerical matching judgments in children with mathematical learning disabilities. Research in Developmental Disabilities, 34, 3182-3189. doi: 10.1016/j.ridd.2013.06.018

Dehaene, S. (1997). The number sense: How the mind creates mathematics. Oxford University Press: Oxford.

Dehaene, S., \& Cohen, L. (1995). Towards an anatomical and functional model of number processing. Mathematical Cognition, 1, 83-120.

Dietrich, J. F., Huber, S., \& Nuerk, H. C. (2015). Methodological aspects to be considered when measuring the approximate number system (ANS) - a research review. Frontiers in Psychology, 17, 295. doi: 10.3389/fpsyg.2015.00295.

Fazio, L. K., Bailey, D. H., Thompson, C. A., \& Siegler, R. S. (2014). Relations of different types of numerical magnitude representations to each other and to mathematics achievement. Journal of Experimental Child Psychology, 123, 5372. doi: 10.1016/j.jecp.2014.01.013 
Gebuis, T., \& Reynvoet, B. (2011). Generating nonsymbolic number stimuli. Behavioral Research Methods, 43, 981-986. doi: 10.3758/s13428-011-0097-5.

Gebuis, T., \& Reynvoet, B. (2012). The interplay between nonsymbolic number and its continuous visual properties. Journal of Experimental Psychology: General, 141, 642-648. doi: 10.1037/a0026218

Gobel, S. M., Watson, S. E., Lervag, A., \& Hulme, C. (2014). Children’s arithmetic development: It is number knowledge, not the approximate number sense, that counts. Psychological Science, 25, 789-798. doi: 10.1177/0956797613516471

Halberda, J., \& Feigenson, L. (2008). Developmental change in the acuity of the “number sense”: The approximate number system in 3-, 4-, 5-, and 6-year-olds and adults. Developmental Psychology, 44, 1457-1465. doi: 10.1037/a0012682

Holloway, I. D., \& Ansari, D. (2009). Mapping numerical magnitudes onto symbols: The numerical distance effect and individual differences in children’s mathematics achievement. Journal of Experimental Child Psychology, 103, 1729. Doi:10.1016/j.jecp.2008.04.001

Hulme, C., Brigstocke, S., \& Moll, K. (2016). Test of Arithmetic and Basic Number Skills (TOBANS). Oxford, England: Oxford University Press.

Hulme, C., \& Snowling, M.J. (2012) Learning to read: What we know and what we need to understand better. Child Development Perspectives, 7, 1-5. doi: 10.1111/cdep.12005

Hulme, C., \& Snowling, M.J. (2013) The interface between spoken and written language: Developmental disorders. Proceedings of the Royal Society of London B, 369, 20120395. doi: 10.1098/rstb.2012.0395. 
Hulme, C., Stothard, S. E., Clarke, P., Bowyer-Crane, C., Harrington, A., Truelove, E., \& Snowling, M. (2009) YARC York Assessment of Reading for Comprehension. Early Reading. GL Publishers.

Hurst, M., Anderson, U., \& Cordes, S. (2017). Mapping among number words, numerals, and nonsymbolic quantities in preschoolers. Journal of Cognition and Development, 18, 41-62. doi: 10.1080/15248372.2016.1228653.

Johansson, B. S. (2007). Numeral writing skill and elementary arithmetic mental calculations. Scandinavian Journal of Educational Research, 49, 3-25. doi: $10.1080 / 0031383042000302119$

Landerl, K., Bevan, A., \& Butterworth, B. (2004). Developmental dyscalculia and basic numerical capacities: A study of 8- to 9-year-old students. Cognition, 93, 99-125. doi: 10.1016/j.cognition.2003.11.004

Leibovich, T., Katzin, N., Harel, M., \& Henik, A. (2017). From “sense of number” to "sense of magnitude": The role of continuous magnitudes in numerical cognition. Behavioral and Brain Sciences, 40, 1-16. doi:10.1017/S0140525X16000960, e164

Libertus, M. E., Odic, D., Feigenson, L., \& Halberda, J. (2016). The precision of mapping between number words and the Approximate Number System predicts children's formal math abilities. Journal of Experimental Psychology, 150, 207-226. doi: 10.1016/j.jecp.2016.06.003.

Litt, R. A., \& Nation, K. (2014). The nature and specificity of paired associate learning deficits in children with dyslexia. Journal of Memory and Language, 71, 71-88. doi: 10.1016/j.jml.2013.10.005

Litt, R. A., de Jong, P. F., van Bergen, E., \& Nation, K. (2013). Dissociating crossmodal and verbal demands in paired associate learning (PAL): What drives 
the PAL-reading relationship? Journal of Experimental Child Psychology, 115, 137-149. doi: 10.1016/j.jecp.2012.11.012

Lyons, I. M., \& Ansari, D. (2009). The cerebral basis of mapping nonsymbolic numerical quantities onto abstract symbols: An fMRI training study. Journal of Cognitive Neuroscience, 21, 1720-1735. doi: 10.1162/jocn.2009.21124.

Lyons, I. M., Bugden, S., Zheng, S., De Jesus, S., \& Ansari, D. (2018). Symbolic number skills predict growth in nonsymbolic number skills in kindergarteners. Developmental Psychology, 54, 440-457. doi: 10.1037/dev0000445

Martin, R. B., Cirino, P. T., Sharp, C., \& Barnes, M. (2014) Number and counting skills in kindergarten as predictors of grade 1 mathematics skills. Learning and Individual Differences, 34, 12-23. doi: 10.1016/j.lindif.2014.05.006.

[dataset] Malone, S. A., Heron-Delaney, M., Burgoyne, K., Hulme, C. Learning correspondences between magnitudes, symbols and words. Open Science Framework, 2016, https://osf.io/ezxb4/

Merkley, R. \& Scerif, G. (2015). Continuous visual properties of number influence the formation of novel symbolic representations. The Quarterly Journal of Experimental Psychology, 68(9), 1860-1870. doi:

10.1080/17470218.2014.994538.

Merkley, R., Shimi, A., \& Scerif, G. (2016). Electrophysiological markers of newly acquired symbolic numerical representations: the role of magnitude and ordinal information. ZDM Mathematics Education, 48, 279-289. doi: 10.1007/s11858015-0751-y 
Mundy, E., \& Gilmore, C. K. (2009). Children’s mapping between symbolic and nonsymbolic representations of number. Journal of Experimental Child Psychology, 103,490-502. doi: 10.1016/j.jecp.2009.02.003

Muthén, L.K., \& Muthén, B.O. (1998-2016). Mplus User’s Guide (6 ${ }^{\text {th }}$ Ed.). Los Angeles, CA: Muthén, L.K., \& Muthén.

Nosworthy N, Bugden S, Archibald L, Evans B, \& Ansari D (2013). A two-minute paper-and-pencil test of symbolic and nonsymbolic numerical magnitude processing explains variability in primary school children's arithmetic competence. PLoS ONE 8(7): e67918. doi:10.1371/journal.pone.0067918

Núñez, R. E. (2017). Is there really an evolved capacity for number? Trends in Cognitive Sciences, 21, 409-424. doi: 10.1016/j.tics.2017.03.005

Pauly, H., Linkersdörfer, J., Lindberg, S., Woerner, W., Hasselhorn, M., \& Lonnemann, J. (2011). Domain-specific rapid automatized naming deficits in children at risk for learning disabilities. Journal of Neurolinguistics, 24, 602610. doi: 10.1016/j.jneuroling.2011.02.002

Piazza, M., \& Dehaene, S. (2004). From number neurons to mental arithmetic: The cognitive neuroscience of number sense. In: Gazzaniga M, editor. The cognitive neurosciences, 3rd edition. New York: Norton; 2004. pp. 865-875.

Piazza, M., Facoetti, A., Trussardi, A. N., Berteletti, I., Conte, S., Lucangeli, D., Dehaene, S., \& Zorzi, M. (2010). Developmental trajectory of number acuity reveals a severe impairment in developmental dyscalculia. Cognition, 116, 3341. doi:10.1016/j.cognition.2010.03.012

Raven, J. C., Court, J. H., \& Raven, J. (1986). Manual for Raven progressive matrices and vocabulary scales. London: Lewis. 
Rousselle, L., \& Noël, M.-P. (2007). Basic numerical skills in children with mathematics learning disabilities: A comparison of symbolic vs non-symbolic number magnitude processing. Cognition, 102, 361-395. doi:10.1016/j.cognition.2006.01.005

Schneider, M., Beeres, K., Coban, L., Merz, S., Schmidt, S. S., Stricker, J., \& de Smedt, B. (2017). Associations of non-symbolic and symbolic numerical magnitude processing with mathematical competence: A meta analysis. Developmental Science, 20, 1-16. doi: 10.1111/desc.12372

van der Sluis, S., de Jong, P. F., \& van der Leij, A. (2004). Inhibition and shifting in children with learning deficits in arithmetic and reading. Journal of Experimental Child Psychology, 87, 239-266.

Verguts, T., \& Fias, W. (2004). Representation of number in animals and humans: A neural model. Journal of Cognitive Neuroscience, 16, 1493-1504. doi:10.1162/0898929042568497.

von Aster, M. G., \& Shalev, R. S. (2007). Number development and developmental dyscalculia. Developmental Medicine and Child Neurology, 49, 868-873. doi: 10.1111/j.1469-8749.2007.00868.x

Warmington, M., \& Hulme, C. (2012). Phoneme awareness, visual-verbal pairedassociate learning, and rapid Automatized naming as predictors of individual differences in reading ability. Scientific Studies of Reading, 16, 45-62. $10.1080 / 10888438.2010 .534832$

Wiese, H. (2003). Numbers, language, and the human mind. Cambridge University Press: Cambridge. 
Xenidou-Dervou, I., Molenaar, D., Ansari, D., van der Schoot, V., \& van Lieshout, E. C. D. M. (2017). Nonsymbolic and symbolic magnitude comparison skills as longitudinal predictors of mathematical achivement. Learning and Instruction, 50, 1-13. doi: 10.1016/j.learninstruc.2016.11.001

Xu, F. \& Spelke, E. S. (2000). Large number discrimination in 6-month-old infants. Cognition, 74, B1-B11. 


\section{Table 1}

Descriptive statistics (Means, Standard Deviations, and Range) and reliabilities for paired associate learning, arithmetic, reading and general cognitive ability tasks

\begin{tabular}{|c|c|c|c|c|c|c|c|}
\hline Variables & $\mathrm{N}$ & Mean (SD) & Reliability & Range & 95\% Confidence-Interval & Skewness & Kurtosis \\
\hline \multicolumn{8}{|l|}{ Paired Associate Learning (PAL) } \\
\hline Verbal-Visual PAL (/20) & 166 & $10.91(4.34)$ & - & $2-20$ & [10.23-11.57] & .16 & -.76 \\
\hline Visual-Verbal PAL (/20) & 166 & $6.80(4.22)$ & - & $0-19$ & [6.15-7.45] & .86 & .65 \\
\hline Magnitude-Visual PAL (/20) & 161 & $9.72(4.58)$ & - & $0-20$ & [9.01- 10.43] & .23 & -.83 \\
\hline Magnitude-Verbal PAL (/20) & 164 & $5.26(4.36)$ & - & $0-20$ & [4.58-5.93] & 1.163 & 1.45 \\
\hline \multicolumn{8}{|l|}{ Arithmetic } \\
\hline Addition (/20) & 164 & $11.04(6.68)$ & .94 & $0-20$ & [10.01-12.07] & -.30 & -1.29 \\
\hline Digit Comparison (/46) & 161 & $14.07(4.64)$ & - & $1-24$ & [13.35-14.80] & -.01 & -.41 \\
\hline \multicolumn{8}{|l|}{ Magnitude Comparison } \\
\hline Ratio .86 (/36) & 163 & $14.64(5.31)$ & - & $0-31$ & [13.82-15.47] & .16 & -.22 \\
\hline Ratio .66 (/36) & 163 & $11.70(4.35)$ & - & $0-24$ & [11.03-12.37] & .04 & .01 \\
\hline Ratio .57 (/36) & 163 & $8.05(2.91)$ & - & $1-16$ & {$[7.60-8.50]$} & .13 & -.11 \\
\hline Digit Writing (/10) & 166 & $3.65(2.59)$ & .46 & $0-10$ & [3.25-4.05] & .33 & -59 \\
\hline \multicolumn{8}{|l|}{ Reading } \\
\hline Reading Fluency Rate & 163 & $.51(.44)$ & - & $-.12-1.7$ & {$[.45-.58]$} & .80 & -.57 \\
\hline Phoneme Deletion (/12) & 163 & $6.60(2.46)$ & .73 & $0-12$ & {$[6.22-6.98]$} & -.17 & -.12 \\
\hline Letter-Sound Knowledge (/17) & 164 & $14.20(2.86)$ & .83 & $3-17$ & [13.76-14.64] & -.86 & .31 \\
\hline RAN Average & 165 & $.81(.22)$ & .92 & $.34-1.32$ & {$[.77-.84]$} & .07 & -.62 \\
\hline Raven’s CPM (/12) & 163 & $7.55(1.59)$ & .31 & $0-12$ & {$[7.30-7.79]$} & -.59 & 2.73 \\
\hline
\end{tabular}

Note: Reliability was assessed using Cronbach’s Alpha; RAN: Rapid Automatized Naming 
Correlations among paired-associate learning tasks, arithmetic, reading and general cognitive ability tasks

\begin{tabular}{|c|c|c|c|c|c|c|c|c|c|c|c|c|c|c|c|}
\hline & 1 & 2 & 3 & 4 & 5 & $\overline{6}$ & 7 & 8 & 9 & 10 & 11 & 12 & 13 & 14 & 15 \\
\hline 1. Reading Fluency & - & $.44^{* *}$ & $.27^{* *}$ & $.52^{* *}$ & $.43^{* *}$ & $.20^{*}$ & $.59 * *$ & $.50^{* *}$ & $.51^{* *}$ & $.40^{* *}$ & $.46^{* *}$ & $.41^{* *}$ & $.35 * *$ & $.31^{* *}$ & .08 \\
\hline 2. Addition & $.65^{* *}$ & - & $.38^{* *}$ & $.37 * *$ & $.31 * *$ & $.30^{* *}$ & $.42 * *$ & $.48^{* *}$ & $.29 * *$ & $.45^{* *}$ & $.45^{* *}$ & $.26^{* *}$ & $.28^{* *}$ & $.18^{*}$ & $.16^{*}$ \\
\hline 3. Magnitude-Visual & $.51 * *$ & $.55^{* *}$ & - & $.25 * *$ & $.40^{* *}$ & $.35 * *$ & $.30 * *$ & $.26 * *$ & $.21^{*}$ & $.31 * *$ & $.34 * *$ & $.24 * *$ & $.26 * *$ & $.27 * *$ & $.27 * *$ \\
\hline 4. Magnitude-Verbal & $.63^{* *}$ & $.50^{* *}$ & $.40^{* *}$ & - & $.45^{* *}$ & $.24 * *$ & $.42^{* *}$ & $.31^{* *}$ & $.29 * *$ & $.21 *$ & $.27 * *$ & $.21^{*}$ & $.22 *$ & .12 & $.19 *$ \\
\hline 5. Visual-Verbal & $.47 * *$ & $.37 * *$ & $.44 * *$ & $.50 * *$ & - & $.39 * *$ & $.36^{* *}$ & $.19 *$ & $.25 * *$ & $.24^{* *}$ & .15 & .15 & .12 & $.23^{* *}$ & $.16^{*}$ \\
\hline 6. Verbal-Visual & $.32 * *$ & $.39 * *$ & $.41^{* *}$ & $.32 * *$ & $.42 * *$ & - & $.23 * *$ & $.25 * *$ & $.18^{*}$ & $.21^{* *}$ & $.21^{* *}$ & .14 & .11 & .06 & .14 \\
\hline 7. Phoneme Deletion & $.72 * *$ & $.61^{* *}$ & $.48^{* *}$ & $.53^{* *}$ & $.41^{* *}$ & $.33 * *$ & - & $.49 * *$ & $.40 * *$ & $.22 * *$ & $.46^{* *}$ & $.31 * *$ & $.32 * *$ & $.21^{*}$ & .02 \\
\hline 8. LSK & $.72 * *$ & $.66^{* *}$ & $.50^{* *}$ & $.48^{* *}$ & $.28^{* *}$ & $.36^{* *}$ & $.66^{* *}$ & - & $.37 * *$ & $.44^{* *}$ & $.43^{* *}$ & $.28^{* *}$ & $.31 * *$ & $.17^{*}$ & .10 \\
\hline 9. RAN Average & $.65^{* *}$ & $.47^{* *}$ & $.40 * *$ & $.43 * *$ & $.32 * *$ & $.28^{* *}$ & $.54 * *$ & $.55 * *$ & - & $.29 * *$ & $.39 * *$ & $.33 * *$ & $.27 * *$ & $.28 * *$ & $.20^{*}$ \\
\hline 10. Digit Writing & $.66^{* *}$ & $.63^{* *}$ & $.53^{* *}$ & $.43^{* *}$ & $.32 * *$ & $.33^{* *}$ & $.48^{* *}$ & $.69 * *$ & $.51^{* *}$ & - & $.40^{* *}$ & $.31^{* *}$ & $.36^{* *}$ & $.28^{* *}$ & .01 \\
\hline 11. Digit Comparison & $.67 * *$ & $.65 * *$ & $.53^{* *}$ & $.44^{* *}$ & $.25^{* *}$ & $.31 * *$ & $.63^{* *}$ & $.64 * *$ & $.55^{* *}$ & $.63^{* *}$ & - & $.54^{* *}$ & $.55^{* *}$ & $.45 * *$ & .15 \\
\hline 12. Magnitude Comparison (ratio .86) & $.58 * *$ & $.48^{* *}$ & $.43^{* *}$ & $.36^{* *}$ & $.22 * *$ & $.23 * *$ & $.50^{* *}$ & $.49^{* *}$ & $.47^{* *}$ & $.52 * *$ & $.69 * *$ & - & $.78^{* *}$ & $.61^{* *}$ & .06 \\
\hline 13. Magnitude Comparison (ratio .66) & $.51^{* *}$ & $.48^{* *}$ & $.44^{* *}$ & $.36^{* *}$ & $.19 * *$ & $.20^{*}$ & $.49 * *$ & $.47^{* *}$ & $.42 * *$ & $.51^{* *}$ & $.66^{* *}$ & $.81^{* *}$ & - & $.51^{* *}$ & .02 \\
\hline 14. Magnitude Comparison (ratio .57) & $.32^{* *}$ & $.24 * *$ & $.31^{* *}$ & 0.15 & $.22 * *$ & 0.10 & $.27 * *$ & $.22^{* *}$ & $.30^{* *}$ & $.29 * *$ & $.45 * *$ & $.60^{* *}$ & $.52 * *$ & - & .01 \\
\hline 15. Raven & $.32 * *$ & $.35 * *$ & $.42 * *$ & $.32 * *$ & $.22 * *$ & $.22 * *$ & $.23 * *$ & $.33^{* *}$ & $.22 * *$ & $.40 * *$ & $.32 * *$ & $.25 * *$ & $.20^{*}$ & 0.08 & - \\
\hline 16. Age & $.65 * *$ & $.57 * *$ & $.50^{* *}$ & $.42 * *$ & $.22 * *$ & $.27 * *$ & $.52^{* *}$ & $.68 * *$ & $.48^{* *}$ & $.69 * *$ & $.59 * *$ & $.48 * *$ & $.44 * *$ & 0.15 & $.40^{* *}$ \\
\hline
\end{tabular}

Notes: Partial correlations controlling for age are above the diagonal. Simple correlations are below the diagonal. LSK=Letter sound knowledge; RAN=Rapid Automatized Naming ; $p<.05, * * p<.01$ 
Table 3

Covariances between predictors in the path model

\begin{tabular}{|c|c|c|c|c|c|c|c|c|c|}
\hline & 1. & 2. & 3. & 4. & 5. & 6. & 7. & 8. & 9. \\
\hline 1. Phoneme deletion & - & & & & & & & & \\
\hline 3. Rapid Automatized & & & & & & & & & \\
\hline Naming & $.36^{* *}$ & $.34 * *$ & - & & & & & & \\
\hline 4. Digit writing & .13 & $.39 * *$ & $.25 * *$ & - & & & & & \\
\hline 5. Digit comparison & $.44^{* *}$ & $.39 * *$ & $.35 * *$ & $.36 * *$ & - & & & & \\
\hline 6. Magnitude comparison & $.30 * *$ & $.27 * *$ & $.30 * *$ & $.34 * *$ & $.63^{* *}$ & & & & \\
\hline 10. Magnitude-visual PAL & $.23 * *$ & $.15^{*}$ & $.15^{*}$ & $.17 *$ & $.26 * *$ & $.24 * *$ & $.31 * *$ & .14 & $.24 * *$ \\
\hline
\end{tabular}

Note. ${ }^{*} \mathrm{p}<.05 * * \mathrm{p}<.01 ;$ as covariances were dropped iteratively some cells above remain blank. 


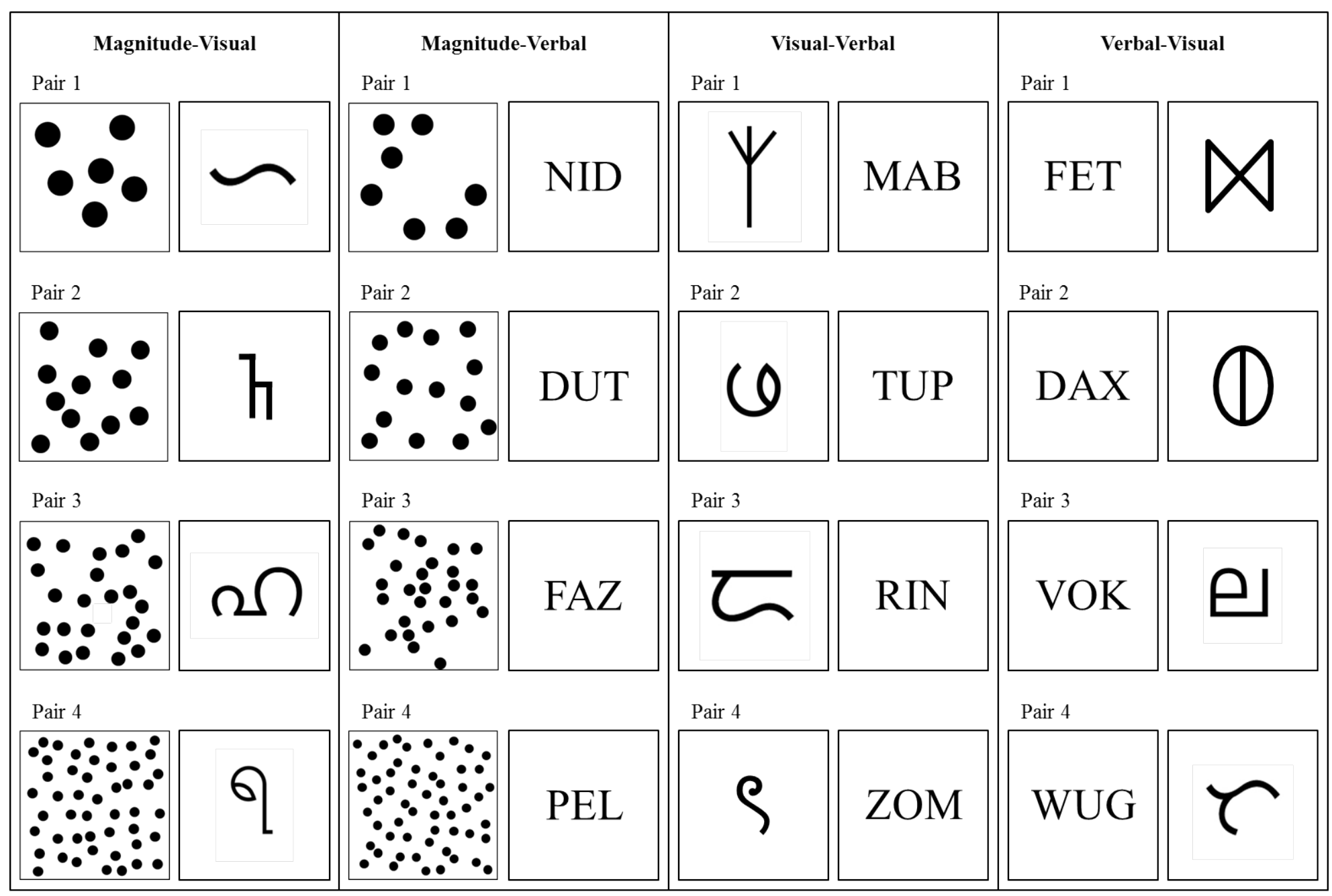

Figure 1. Stimulus pairs presented to children in the PAL tasks. 


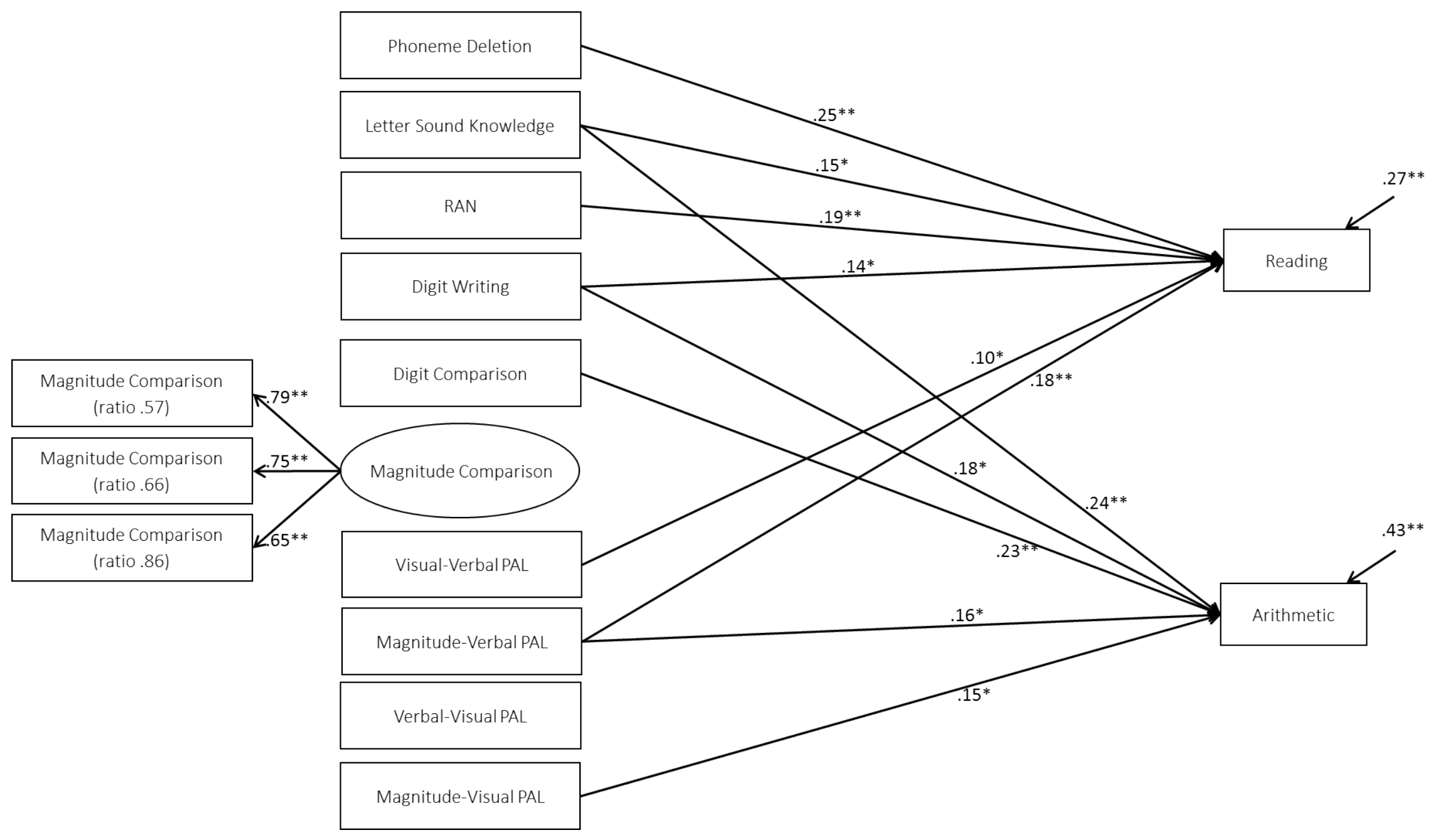

Figure 2. Path model predicting arithmetic and reading performance in children. ${ }^{*} p<.05,{ }^{* *} p<.001$ 